\title{
A NOTE ON INTEGER SOLUTIONS OF THE DIOPHANTINE EQUATION $x^{2}-d y^{2}=1$
}

\author{
by JOHN HUNTER
}

(Received lst June, 1956)

In the equation

$$
x^{2}-d y^{2}=1,
$$

$d$ is any positive integer which is not a perfect square. For convenience we shall consider only those solutions of (1) for which $x$ and $y$ are both positive. All the others can be obtained from these. In fact, it is well known that if $\left(x_{0}, y_{0}\right)$ is the minimum positive integer solution of (1), then all integer solutions $(x, y)$ are given by

$$
x+y \sqrt{ } d= \pm\left(x_{0}+y_{0} \sqrt{ } d\right)^{n} \quad(n=0, \pm 1, \pm 2, \ldots),
$$

and, in particular, all positive integer solutions are given by

$$
x+y \sqrt{ } d=\left(x_{0}+y_{0} \sqrt{ } d\right)^{n} \quad(n=1,2,3, \ldots) .
$$

The purpose of this note is to establish a procedure for obtaining solutions of (1) by Newton's method of approximating to the root $\sqrt{ } d$ of the equation $f(x) \equiv x^{2}-d=0$.

If $x_{1}$ is any positive (for convenience) rational number, then Newton's method gives a sequence $\left(x_{n}\right)$ of rational numbers which is defined by the relation

$$
x_{n+1}=x_{n}-\frac{f\left(x_{n}\right)}{f^{\prime}\left(x_{n}\right)}=x_{n}-\frac{x_{n}^{2}-d}{2 x_{n}}=\frac{x_{n}^{2}+d}{2 x_{n}} \quad(n \geqslant 1),
$$

and which converges to $\sqrt{ } d$. We first note that if (2) is written in the form $x_{n+1}=p_{n+1} / q_{n+1}$, where $p_{n+1}$ and $q_{n+1}$ are the positive rational numbers defined by

$$
p_{n+1}=\frac{x_{n}^{2}+d}{\left|x_{n}^{2}-d\right|}, \quad q_{n+1}=\frac{2 x_{n}}{\left|x_{n}^{2}-d\right|},
$$

then it is easily verified that $\left(p_{n+1}, q_{n+1}\right)$ is a rational solution of (1) for $n=1,2,3, \ldots$. For $n \geqslant 2$, since $x_{n}=p_{n} / q_{n}$ and $p_{n}^{2}-d q_{n}^{2}=1$, (3) can be simplified to

$$
p_{n+1}=p_{n}^{2}+d q_{n}^{2}, \quad q_{n+1}=2 p_{n} q_{n} .
$$

Thus, corresponding to each positive rational number $x_{1},(2)$ provides a sequence of positive rational solutions $\left(p_{n}, q_{n}\right)(n=2,3,4, \ldots)$ of $(1)$ which, by (4), are such that

$$
p_{n+1}+q_{n+1} \sqrt{ } d=\left(p_{n}+q_{n} \sqrt{ } d\right)^{2},
$$

and hence, by induction, such that

$$
\begin{gathered}
p_{n+1}+q_{n+1} \sqrt{ } d=\left(p_{2}+q_{2} \sqrt{ } d\right)^{2^{n-1}}, \\
p_{2}=\frac{x_{1}^{2}+d}{\left|x_{1}^{2}-d\right|}, \quad q_{2}=\frac{2 x_{1}}{\left|x_{1}^{2}-d\right|} .
\end{gathered}
$$

Also, if $p_{2}$ and $q_{2}$ are integers, then (5) gives a sequence of positive integer solutions of (1).

We now establish some properties of the method in the following lemma.

LEMMA. (i) $p_{2}$ and $q_{2}$ are integers if and only if $x_{1}^{2}-d$ divides $2 x_{1}$.

(ii) If $(p, q)$ is a given positive rational solution of $(1)$, then there are two values of $x_{1}, y_{1}$ and $y_{1}^{*}$ say, which give rise, by (6), to the solution $(p, q)$, and these have the properties

$$
y_{1}>\sqrt{ } d . \quad y_{1}^{*}<\sqrt{ } d, \quad y_{1} y_{1}^{*}=d, \quad y_{1}-y_{1}^{*}=2 / q .
$$


Proof. (i) If $p_{2}$ and $q_{2}$ are integers, then $x_{1}^{2}-d$ divides $2 x_{1}$. Conversely, if. $x_{1}^{2}-d$ divides $2 x_{1}$, then $q_{2}$ is an integer and, since $\frac{x_{1}^{2}+d}{x_{1}^{2}-d}=1+\frac{2 d}{x_{1}^{2}-d}$ and $\left(x_{1}^{2}-d, x_{1}\right)$ divides $d$, it follows that $p_{2}$ is also an integer.

(ii) We have to determine the values of $x_{1}$ for which $p=\frac{x_{1}^{2}+d}{\left|x_{1}^{2}-d\right|}$ and $q=\frac{2 x_{1}}{\left|x_{1}^{2}-d\right|}$. Suppose first that $x_{1}^{2}>d$; then $p=\frac{x_{1}^{2}+d}{x_{1}^{2}-d}, q=\frac{2 x_{1}}{x_{1}^{2}-d}$ and hence $x_{1}^{2}=\frac{p+1}{p-1} d=\frac{2 x_{1}+q d}{q}$, so that $x_{1}=\frac{q d}{p-1}$. Similarly, if $x_{1}^{2}<d$, then $p=\frac{x_{1}^{2}+d}{d-x_{1}^{2}}, q=\frac{2 x_{1}}{d-x_{1}^{2}}$ and $x_{1}=\frac{q d}{p+1}$. If these values of $x_{1}$ are denoted by $y_{1}$ and $y_{1}^{*}$, respectively, then $y_{1}>\sqrt{ } d, y_{1}^{*}<\sqrt{ } d, y_{1} y_{1}^{*}=\frac{q^{2} d^{2}}{p^{2}-1}=d$, since $p^{2}-d q^{2}=1$, and $y_{1}-y_{1}^{*}=\frac{2 q d}{p^{2}-1}=\frac{2}{q}$.

From this last equation it follows that if $y_{1}$ and $y_{1}^{*}$ are integers, then $q=1$ or 2 , and that if $q \geqslant 3$, then $y_{1}$ and $y_{1}^{*}$ are not both integers.

We now show that for certain values of $d$ a corresponding easily-determined value of $x_{1}$ gives rise, by (6), to the minimum positive integer solution of (1). Since $d$ is a positive integer which is not a perfect square, it can be expressed uniquely in the form $d=m^{2}+r$ where $r \neq 0,-m+1 \leqslant r \leqslant m$, for some positive integer $m$.

Theorem. (i) If $r=-1$, then $x_{1}=m-1$ gives the minimum positive integer solution of (1), and this is $(m, 1)$.

(ii) If $r$ divides $2 m, r \neq-1$, then $x_{1}=m$ gives the minimum positive integer solution of (1), and this is $\left(\frac{2 m^{2}+r}{|r|}, \frac{2 m}{|r|}\right)$.

Proof. (i) In this case, $d-x_{1}^{2}=2(m-1), d+x_{1}^{2}=2 m(m-1)$ and, by $(6), p_{2}=m, q_{2}=1$, and this is clearly the minimum positive integer solution.

(ii) Here $\left|x_{1}^{3}-d\right|=|r|$, so that $x_{1}^{2}-d$ divides $2 x_{1}$ and thus, by part (i) of the above lemma, $p_{2}$ and $q_{2}$ are integers. From (6), $p_{2}=\frac{2 m^{2}+r}{|r|}$ and $q_{2}=\frac{2 m}{|r|}$. Suppose now that $\left(x_{0}, y_{0}\right)$ is the minimum positive integer solution of (1). Then the second minimum positive integer solution is $\left(x_{0}^{2}+d y_{0}^{2}, 2 x_{0} y_{0}\right)$, that is $\left(1+2 d y_{0}^{2}, 2 x_{0} y_{0}\right)$. Now $1+2 d y_{0}^{2} \geqslant 1+2 d$; but

$$
\frac{2 m^{2}+r}{|r|}=\frac{2 d-r}{|r|} \leqslant \frac{2}{|r|} d+1<2 d+1 \text { if }|r|>1,
$$

and also

$$
\frac{2 m^{2}+r}{|r|}=2 d-1<2 d+1 \text { if } r=1 \text {. }
$$

Hence the positive integer solution $\left(p_{2}, q_{2}\right)$ is the minimum positive integer solution.

It is of interest to note that the results of this theorem give the minimum positive integer solutions of (1) for all values of $d$ in the range $2 \leqslant d \leqslant 40$ except for $d=13,19,21,22,28,29$ and 31.

We note finally that the results in the theorem are not new (see, for example, L. E. Dickson's History of the theory of numbers, II, p. 378). It is surprising that no account of the relation of Newton's method to the equation $x^{2}-d y^{2}=1$ appears to exist in the literature.

The UnIversity

GLasGow 